\title{
Identification of immunity-related genes in prostate cancer and potential role of the ETS family of transcription factors in their regulation
}

\author{
DAVID ADLER ${ }^{1}$, ANDREAS LINDSTROT $^{1}$, JÖRG ELLINGER $^{2}$, SEBASTIAN ROGENHOFER $^{2}$, \\ REINHARD BUETTNER $^{1}$ and NICOLAS WERNERT ${ }^{1}$ \\ ${ }^{1}$ Institute of Pathology and ${ }^{2}$ Department of Urology, University Hospital Bonn, D-53127 Bonn, Germany
}

Received May 30, 2011; Accepted July 5, 2011

DOI: $10.3892 /$ ijmm.2011.771

\begin{abstract}
The role of the immune response in tumor progression, and disease outcome is still debated, and a lack of knowledge of the immune defenses in prostate cancer still exists. In addition, the ETS family of transcription factors which is involved in translocations frequently found in prostate cancer is reported to be essential for the regulation of immunity-related genes. In order to identify immunity-related genes in prostate cancer, we performed two microarrays using RNA extracted from laser microdissected glands of the normal prostate proper (or the peripheral zone) and moderately and poorly differentiated prostate carcinomas from patients who had undergone radical prostatectomy. Many differentially expressed genes were found, however, only immunity-related genes (B cell, innate, and $\mathrm{T}$ cell immunity) with an expression of more than 10 -fold increase or decrease and a $\mathrm{P}<0.01$ between the moderately differentiated tumors and the normal glands, and the poorly differentiated tumors and the normal glands were considered significant. Based on these two microarrays, we identified a set of 37 genes that were up- or down-regulated in tumors (moderately and poorly differentiated) compared to the normal glands. Analysis of these genes revealed, strikingly, that $31 / 37$ of these genes have potential binding sites within their promoter regions for members of the ETS family of transcription factors, and some are reported to be targets of ETS members. These findings identified immunity-related genes in prostate cancer, and provided insights into their potential regulation, which may lead to a better early detection, immunotherapy, and therapeutic drug treatment of this disease. Unraveling the dynamics of the ETS-immunity-related genes will provide an invaluable insight into understanding prostate cancer immunology.
\end{abstract}

Correspondence to: Professor Nicolas Wernert, Institute of Pathology, University Hospital Bonn, Sigmund-Freud-Str. 25, D-53127 Bonn, Germany

E-mail: nicolas.wernert@ukb.uni-bonn.de

Key words: ETS, transcriptional regulation, prostate, immunity, innate, B cell, $\mathrm{T}$ cell

\section{Introduction}

Prostate cancer is a clinically heterogeneous-multifocal disease with a clinical outcome difficult to predict, and one of the most prevalent malignancies in men (1-4). The role the host immune response plays in tumor progression is still in dispute, and studies have suggested that the immune system can promote or suppress tumor growth (5). It has been suggested that production of cytokines by the activation of innate immunity and inflammation can either stimulate or inhibit tumor growth and progression (6). The majority of pro-inflammatory cytokines produced by the host immune cells or tumor cells promote tumor development, whereas pro-apoptotic and anti-inflammatory cytokines usually interfere with tumor development (6). A weaker host-mediated antitumor activity than a tumor-mediated immunosuppressive activity, leads to immune escape and rapid growth of the tumor cells, whereas a stronger antitumor activity leads to the eradication of the tumor cells (6).

During the progress of cancer, many patients will develop innate and adaptive immunity responses (5). In prostate cancer, a combination of TH1-adaptive immunity and inflammation genes has been suggested to confer improved prognosis (5). A role of the immune system in determining disease outcome, and an association between the body's immune system and tumor behavior have also been suggested (5). The prostate is relatively predisposed to several degrees of inflammation, and inflammation is suggested to contribute to prostatic carcinogenesis $(7,8)$. The reasons that lie behind such a predisposition are still unclear; however the ability of the body's immune response against prostate tissues is undeniable (7). A recent study using a mouse model, suggested that $\mathrm{T}$ regulatory lymphocytes down-regulated inflammatory cytokines, and that inflammatory cytokines are significantly elevated and linked with prostate cancer development, but without the presence of inflammatory disease in the prostate (9).

It has been reported that immunity against normal or cancerous prostate tissues can be induced by manipulating the immune system (7). Stimulating T-cell responses is the most immunotherapeutic approach in prostate cancer (7). Reduced infiltration of tumor-associated macrophages is associated with prostate cancer progression (10). Furthermore, absence 
or weak tumor infiltrating lymphocytes are associated with high risk of tumor progression (11). Studies reported that an immune response in the form of autoantibodies against certain tumor antigens develops in many cancer patients (12-15), therefore subsequent studies attempted to use these autoantibody signatures to measure the immune response in prostate cancer and utilize it as a possible early detection method for the disease (15).

Prostate cancer can avoid the immune defenses by presenting a defective antigen, expressing immunosuppressive molecules, $\mathrm{T}$ cell receptor dysfunction, and active immune down-modulation (16). Most prostate cancer patients are immunocompetent, even though the tumor avoidance mechanisms of the immune defenses alter the host immunity (16). The failure of the immune defenses against the tumor can be interpreted as an immunological tolerance (16). Therefore, identifying and understanding the regulation of immunity-related genes in prostate cancer is of a great importance to be able to counter this disease.

The ETS family of transcription factors which is involved in translocations frequently found in prostate cancer (eg. TMPRSS2-ERG fusions $50-80 \%$ of occurrences, TMPRSS2-ETV-1 $20 \%$ of occurrences) $(17,18)$ is suggested to be essential for the regulation of the immune system, and the regulation of immunity-related genes (19). The ETS family of transcription factors has 27 members in humans and is characterized by an evolutionary highly conserved DNA-binding domain, the ETS domain, which consists of 80 amino acids with 4 tryptophan repeats $(20,21)$. This common ETS domain interacts with nucleotide sequences that are centred around a GGAA/T motif (19). Binding preferences for distinct sequences flanking the GGAA/T motif facilitate specificity of the individual ETS-factor binding and may explain the unique biological functions of the different ETS factors $(22,23)$. The ETS family is involved in various biological processes, such as development, differentiation, proliferation, apoptosis, migration, tissue remodelling, invasion and angiogenesis in a variety of cell types such as B cells, endothelial cells, fibroblasts as well as neoplastic cells (24-28).

The ETS family members ETS1, ETS2, ELF1, ELF3, ELF4, FLI1, GABP, SPI1 and SPIB are reported to regulate genes involved in immunity (19). While these ETS members usually activate transcription of pathogen and tumor defense-associated genes, some also repress gene transcription (19). Notably, ETS1, the family prototype and SPI1 seem to have the highest effect on immunity, primarily, by controlling immune cell development (19), whereas, other members such as ELF1 and FLI1 are implicated in autoimmunity, indicating that ETS factors can be negative regulators of immunity as well as positive ones (19).

Immunity-related genes regulated by ETS family members and their roles in immunity have been described in detail in a previous review (19). The ETS factors are reported to play roles in activation as well as repression of immunity-related genes (19). For instance, genes such as the ones encoding TCR $\alpha$ which facilitates $\mathrm{T}$ cell response to peptides presented on $\mathrm{MHC}$, and $\mathrm{CD} 3 \delta$ which is involved in T cell activation are activated by ETS1, whereas, genes such as the ones encoding TCR $\beta$ which is involved in T cell response, and IL2 which promotes lymphocyte proliferation, are repressed by ETS1 (19). Notably, the $c$-fms gene for instance which is involved in macrophage activation, can be activated by ETS1, as well as
ETS2 and SPI1, while the gene encoding IL5, which promotes development and activity of eosinophil granulocytes, can be activated in cooperation with other factors by ETS1 and ETS2 (19). Other genes which can also be activated by multiple ETS members include, the $\mathrm{mbl}$ gene which is involved in facilitating B cell antigen receptor trafficking and signal transduction, and the IL3 gene which is involved in promoting lymphoid and myeloid growth, by GABP $\alpha$ / FLI1, and ELF1/ELF4, respectively (19). Other examples include the activation of the genes CD49D and CD18 which are involved in facilitating leukocyte migration, by GABP $\alpha$, and the genes encoding IL18/IL1 $\beta$ which promotes NK and Th1 cell activity and NK cell activation, respectively, by ELF3 (19).

In this study, we report the identification of immunityrelated genes (B cell, innate, and $\mathrm{T}$ cell immunity) in prostate cancer, and their potential regulation by members of the ETS family of transcription factors. Identification of these immunity-related genes may have great implications into our understanding of prostate cancer immunology, and potentially lead to a better early detection, immunotherapy, and therapeutic drug treatment of this disease.

\section{Materials and methods}

Ethics statement. This study has been approved by the Faculty of Medicine's ethics review board of the University of Bonn/ University Hospital, Germany, according to the principles expressed in the Declaration of Helsinki. Furthermore, written informed consents were obtained from all participants involved in the study, and were approved by the Faculty of Medicine's ethics review board of the University of Bonn/University Hospital.

Microarray raw data. The microarray raw data have been depostied at GEO under the accession number GSE28615 and we can confirm that all details are MIAME compliant.

Processing of human prostatectomy specimens. Prostate specimens were obtained from prostate carcinoma patients immediately after radical prostatectomy. The age of the patients ranged from 45-83 years (average of 67.1 years). Fresh tissue samples $(0.5 \times 0.5 \times 0.3 \mathrm{~cm})$ were taken out of the peripheral zones (prostate proper) of 5 moderately differentiated (Gleason-scores 6 and 7a) prostate carcinoma patients, 4 poorly differentiated (Gleason-scores 8 and 9) prostate carcinomas patients, as well as 10 normal peripheral zones of prostate cancer patients. The tissues were then shockfrozen in liquid nitrogen with ice-cold isopentane. Frozen sections of $6 \mu \mathrm{m}$ were cut from the samples using a cryotome (LEICA, Germany) and mounted on membrane-coated slides (Membrane Slides, $1 \mathrm{~mm}$ PEN, Zeiss, Germany) for subsequent laser-microdissection. One section was mounted on conventional slides and stained with hematoxylin and eosin (H\&E) for diagnostic evaluation by an experienced pathologist. Laser-microdissection was performed as previously described (29-31). Frozen sections were dried for $2 \mathrm{~min}$ in the cryotome, washed for 2 min with 70\% ethanol in DEPCtreated water and stained for $30 \mathrm{sec}$ in $1 \%$ Cresyl Violet diluted in 50\% ethanol-DEPC-treated water. Slides were then washed briefly in 70 and $100 \%$ ethanol, dried for $10 \mathrm{~min}$ and stored at 
Table I. Poorly differentiated tumors vs. normal glands of the peripheral zone: B cell immunity genes (up-regulated).

\begin{tabular}{llc}
\hline Short name & \multicolumn{1}{c}{ Long name } & Fold change \\
\hline HDAC9 & Histone deacetylase 9 & 11.7 \\
CDKN2A & Cyclin-dependent kinase inhibitor 2A & \\
& (melanoma, p16, inhibits CDK4) & 10.1 \\
SH2D1A & SH2 domain protein 1A & 10.1 \\
\hline
\end{tabular}

Table II. Moderately differentiated tumors vs. normal glands of the peripheral zone: B cell immunity genes (up-regulated).

\begin{tabular}{llc}
\hline Short name & \multicolumn{1}{c}{ Long name } & Fold change \\
\hline SH2D1A & $\begin{array}{l}\text { SH2 domain protein 1A } \\
\text { IL5 }\end{array}$ & $\begin{array}{l}\text { Interleukin 5 (colony-stimulating } \\
\text { factor, eosinophil) }\end{array}$ \\
& & 14.9 \\
\hline
\end{tabular}

$-80^{\circ} \mathrm{C}$ until use for laser-microdissection of normal glands and the stroma between them.

Quality control. The quality of the RNA was measured from every patient before laser microdissection using the laser capture microscope (LCM). The section was washed from the slide with $600 \mu \mathrm{l}$ buffer RLT $+2 \mu \mathrm{M}$ DTT (provided by the kit RNeasy mini kit, Qiagen, Germany) and vortexed for $30 \mathrm{sec}$. RNA-extraction was performed as described by the manufacturer. The recommended DNase digestion was made with the RNase-free DNase Set (Qiagen). The quality of the RNA was measured with the Agilent Bioanalyzer 2100 (Agilent Technologies, USA). Samples with a RIN factor $>6$ were used for LCM.

Laser-capture microscopy. The cresyl violet stained sections were cut with an Axio Observer Z1 Microscope (Zeiss, Germany) and installed PALM MicroBeam (Zeiss). The LCM was performed under 10X objective. The glands from carcinomas and the normal peripheral zones were isolated from the stroma by laser microdissection, and collected in $200 \mu \mathrm{l}$ Adhesive Cap tubes (Zeiss).

Microarray analysis of RNA isolated from laser microdisected moderately and poorly differentiated prostate carcinoma glands, as well as from normal glands of prostate cancer patients. RNA was isolated from laser microdisected moderately and poorly differentiated prostate carcinoma glands, as well as from normal glands from prostate carcinoma patients using the RNeasy micro kit (Qiagen) as described by the manufacturer. The recommend DNase digestion was included with the RNase-free DNase Set. The amount of the isolated RNA was measured with the NanoDrop photometer (Thermo Fisher Scientific, USA). Afterwards, an equal amount of RNA from the normal peripheral glands, moderately differentiated glands, and poorly differentiated glands were pooled to final concentrations of $300 \mathrm{ng}$ of RNA, respectively. These pools were sent to Miltenyi Biotec (Bergisch Gladbach, Germany) for the microarray analysis and bioinformatical interpretation.
Table III. Poorly differentiated tumors vs. normal glands of the peripheral zone: innate immunity genes (up-regulated).

\begin{tabular}{llc}
\hline Short name & \multicolumn{1}{c}{ Long name } & Fold change \\
\hline CRISP3 & Cysteine-rich secretory protein 3 & 43.6 \\
CXCL11 & Chemokine (C-X-C motif) ligand 11 & 21.3 \\
ADORA1 & Adenosine A1 receptor & 17.5 \\
CXCL9 & Chemokine (C-X-C motif) ligand 9 & 15 \\
CXCL10 & Chemokine (C-X-C motif) ligand 10 & 14 \\
IDO1 & Indoleamine 2,3-dioxygenase 1 & 13.4 \\
IL2RA & Interleukin 2 receptor, $\alpha$ & 12.1 \\
HDAC9 & Histone deacetylase 9 & 11.7 \\
SH2D1A & SH2 domain protein 1A & 10.1 \\
\hline
\end{tabular}

The RNA was labeled with Cy3 and hybridized on the Whole Human Genome Oligo Microarray 4x44K (Agilent) according to the manufacturer's instructions. The microarray results were then validated by qRT-PCR of a subset of genes.

Transcriptional regulatory element database (TRED). The TRED database was used to determine whether members of the ETS family of transcription factors are known to target any of the 37 genes found (32). Hence, each gene was entered into the TRED database and a list of reported regulators was retrieved.

Transcription factor search (TFSEARCH). Search for ETS transcription factor binding sites within the promoter regions of the 37 genes was performed using the TFSEARCH $(33,34)$. The promoter retrieval of each gene was performed using the TRED database (32), followed by insertion into the TFSEARCH database $(33,34)$.

\section{Results}

Gene expression analysis of moderately and poorly differentiated prostate carcinoma glands compared to normal glands of the prostate proper (or the peripheral zone) from prostate cancer patients using Whole Human Genome Oligo microarrays. To identify immunity-related genes in prostate cancer, we analyzed the expression profile of genes involved in innate, $\mathrm{B}$ cell, and $\mathrm{T}$ cell immunity in the moderately and poorly differentiated prostate carcinoma glands compared to normal glands of the prostate proper (or the peripheral zone) from prostate cancer patients using two Whole Human Genome Oligo microarrays. Genes with an expression of $>10$-fold increase or decrease and a $\mathrm{P}<0.01$ were considered significant and were selected for further analysis. A clustering analysis based on gene function placed each gene into an immunity-related category: B cell, innate, and/or T cell immunity.

Genes involved in B cell immunity. Genes found to be $>10$-fold up or down-regulated, with a $\mathrm{P}<0.01$, and involved in B cell immunity are shown in Tables I, II, VII and VIII. The genes HDAC9, CDKN2A, SH2D1A and IL5 were found to be up-regulated in either the moderately or poorly differentiated prostate carcinoma glands or in both compared to the normal glands (Tables I and II). On the contrary, LTF, DMBT1, CD38, 
Table IV. Moderately differentiated tumors vs. normal glands of the peripheral zone: innate immunity genes (up-regulated).

\begin{tabular}{llc}
\hline Short name & \multicolumn{1}{c}{ Long name } & Fold change \\
\hline PGLYRP1 & Peptidoglycan recognition protein 1 & 100 \\
CRISP3 & Cysteine-rich secretory protein 3 & 56.9 \\
ALOX15 & Arachidonate 15-lipoxygenase & 21.5 \\
FGR & Gardner-Rasheed feline sarcoma & \\
& viral (v-fgr) oncogene homolog & 15.2 \\
SH2D1A & SH2 domain protein 1A & 14.9 \\
CXCL11 & Chemokine (C-X-C motif) ligand 11 & 13.8 \\
ADORA1 & Adenosine A1 receptor & 13.1 \\
IDO1 & Indoleamine 2,3-dioxygenase 1 & 12.3 \\
IL5 & Interleukin 5 (colony-stimulating & \\
& factor, eosinophil) & 11.7 \\
\hline
\end{tabular}

Table V. Poorly differentiated tumors vs. normal glands of the peripheral zone: $\mathrm{T}$ cell immunity genes (up-regulated).

\begin{tabular}{llc}
\hline Short name & \multicolumn{1}{c}{ Long name } & Fold change \\
\hline IDO1 & Indoleamine 2,3-dioxygenase 1 & 13.4 \\
IL2RA & Interleukin 2 receptor, & 12.1 \\
CDKN2A & Cyclin-dependent kinase inhibitor 2A & \\
& (melanoma, p16, inhibits CDK4) & 10.1 \\
\hline
\end{tabular}

Table VI. Moderately differentiated tumors vs. normal glands of the peripheral zone: $\mathrm{T}$ cell immunity genes (up-regulated).

\begin{tabular}{lcc} 
Short name & Long name & Fold change \\
\hline IDO1 & Indoleamine 2,3-dioxygenase 1 & 12.3 \\
\hline
\end{tabular}

Table VII. Poorly differentiated tumors vs. normal glands of the peripheral zone: B cell immunity genes (down-regulated).

\begin{tabular}{llc}
\hline Short name & \multicolumn{1}{c}{ Long name } & Fold change \\
\hline LTF & Lactotransferrin & -100 \\
DMBT1 & Deleted in malignant brain tumors 1 & -27.2 \\
CD38 & CD38 molecule & -22.1 \\
BCL11A & B-cell CLL/lymphoma 11A & \\
& (zinc finger protein) & -10.9 \\
\hline
\end{tabular}

BCL11A and ITGA4 were down-regulated in either the moderately or poorly differentiated prostate carcinoma glands or in both compared to the normal glands (Tables VI and VIII).

Genes involved in innate immunity. Genes that were found to be $>10$-fold up and down-regulated, with a $\mathrm{P}<0.01$ and involved in innate immunity are shown in Tables III, IV, IX and X. Among these genes, CRISP3, CXCL11, ADORA1, CXCL9, CXCL10,
Table VIII. Moderately differentiated tumors vs. normal glands of the peripheral zone: B cell immunity genes (down-regulated).

\begin{tabular}{llc}
\hline Short name & \multicolumn{1}{c}{ Long name } & Fold change \\
\hline LTF & Lactotransferrin & -13.4 \\
ITGA4 & $\begin{array}{l}\text { Integrin, } \alpha \text { 4 (antigen CD49D, } \\
\alpha \text { 4 subunit of VLA-4 receptor) }\end{array}$ & -12.2 \\
\hline
\end{tabular}

Table IX. Poorly differentiated tumors vs. normal glands of the peripheral zone: innate immunity genes (down-regulated).

\begin{tabular}{llc}
\hline Short name & \multicolumn{1}{c}{ Long name } & Fold change \\
\hline LTF & Lactotransferrin & -100 \\
CXCL2 & Chemokine (C-X-C motif) ligand 2 & -87.9 \\
ORM1 & Orosomucoid 1 & -72.5 \\
CCK & Cholecystokinin & -60.5 \\
CXCL3 & Chemokine (C-X-C motif) ligand 3 & -36.6 \\
ORM2 & Orosomucoid 2 & -36.5 \\
DMBT1 & Deleted in malignant brain tumors 1 & -27.2 \\
SAA1 & Serum amyloid A1; serum amyloid A2 & -24.6 \\
C5AR1 & Complement component 5a receptor 1 & -23.8 \\
SERPINA3 & Serpin peptidase inhibitor, clade A ( $\alpha-1$ & \\
& antiproteinase, antitrypsin), member 3 & -21.9 \\
SCGB1A1 & Secretoglobin, family 1A, member 1 & \\
& (uteroglobin) & -19.6 \\
CXCL6 & Chemokine (C-X-C motif) ligand 6 & \\
& (granulocyte chemotactic protein 2) & -18.3 \\
SAA4 & Serum amyloid A4, constitutive & -17.4 \\
CXCL1 & Chemokine (C-X-C motif) ligand 1 & \\
& (melanoma growth stimulating activity, $\alpha)$ & -16.5 \\
NOS2 & Nitric oxide synthase 2, inducible & -15.5 \\
NOX1 & NADPH oxidase 1 & -10.7 \\
AOX1 & Aldehyde oxidase 1 & -10.3 \\
\hline & &
\end{tabular}

IDO1, IL2RA, HDAC9, SH2D1A, PGLYRP1, ALOX15, FGR and $I L 5$ were found to be up-regulated in either the moderately or poorly differentiated prostate carcinoma glands or in both compared to the normal glands (Tables III and IV). On the other hand, LTF, CXCL2, ORM1, CCK, CXCL3, ORM2, DMBT1, SAA1, C5AR1, SERPINA3, SCGB1A1, CXCL6, $S A A 4, C X C L 1, N O S 2, N O X 1, A O X 1, A C E 2$ and $A B C C 9$ were found to be down-regulated in either the moderately or poorly differentiated prostate carcinoma glands or in both compared to the normal glands (Tables IX and X).

Genes involved T cell immunity. Genes that were $>10$-fold up or down-regulated, with a $\mathrm{P}<0.01$ and involved in T cell immunity are shown in Tables V, VI, XI and XII. The genes IDOI, IL2RA and $C D K N 2 A$ were found up-regulated in either the moderately or poorly differentiated prostate carcinoma glands or in both compared to the normal glands (Tables V and VI), whereas SCGB1A1 and BCL11A were found to be down-regulated in the poorly differentiated, and $P R L R$ down-regulated in the moderately differentiated carcinoma glands, compared to the normal glands, respectively (Tables XI and XII). 
Table X. Moderately differentiated tumors vs. normal glands of the peripheral zone: innate immunity genes (down-regulated).

\begin{tabular}{lll}
\hline Short name & \multicolumn{1}{c}{ Long name } & Fold change \\
\hline C5AR1 & Complement component 5a receptor 1 & -41.8 \\
$A C E 2$ & $\begin{array}{l}\text { Angiotensin I converting enzyme } \\
\text { (peptidyl-dipeptidase A) 2 }\end{array}$ & -33.6 \\
& Aldehyde oxidase 1 & -14.7 \\
AOX1 & Lactotransferrin & -13.4 \\
$A B C C 9$ & ATP-binding cassette, sub-family C & \\
& (CFTR/MRP), member 9 & -11.3 \\
$C C K$ & Cholecystokinin & -11.2 \\
\hline
\end{tabular}

Table XI. Poorly differentiated tumors vs. normal glands of the peripheral zone: $\mathrm{T}$ cell immunity genes (down-regulated).

\begin{tabular}{llc}
\hline Short name & \multicolumn{1}{c}{ Long name } & Fold change \\
\hline SCGB1A1 & $\begin{array}{l}\text { Secretoglobin, family 1A, } \\
\text { member 1 (uteroglobin) }\end{array}$ & -19.6 \\
BCL11A & $\begin{array}{l}\text { B-cell CLL/lymphoma 11A } \\
\text { (zinc finger protein) }\end{array}$ & -10.9 \\
\hline
\end{tabular}

Table XII. Moderately differentiated tumors vs. normal glands of the peripheral zone: $\mathrm{T}$ cell immunity genes (down-regulated).

\begin{tabular}{lcc}
\hline Short name & Long name & Fold change \\
\hline$P R L R$ & Prolactin receptor & -100 \\
\hline
\end{tabular}

Genes with ETS transcription factors binding sites within their promoter regions. Search for ETS transcription factors binding sites within the promoter regions of the 37 genes using TFSEARCH revealed that $31 / 37$ of these genes have potential binding sites for members of the ETS family of transcription factors (Table XIII).

Genes reported to be targets of ETS family members. Using the TRED database (32), we retrieved a list of 5/31 genes that were reported to be targets by members of the ETS family of transcription factors $(19,32,35-38)$ (Table XIV).

\section{Discussion}

The role of the host immune response in tumor progression is still in dispute (5). The immune system has been suggested to promote or suppress tumor growth, and to play a role in determining disease outcome (5). It has also been suggested that there is an association between the body's immune system and tumor behavior (5). In prostate cancer, the majority of patients are immunocompetent, in spite of alterations of the host immunity by the tumor avoidance mechanisms of the immune defenses (16). The immune defense's failure to act against the tumor can be interpreted as an immunological tolerance (16).
Table XIII. Genes with potential ETS transcription factors binding sites within their promoter regions.

\begin{tabular}{|c|c|}
\hline Gene name & Potential ETS family members \\
\hline$A B C C 9$ & ETS1 \\
\hline$A D O R A 1$ & ETS1 \\
\hline$A O X 1$ & ETS1 \\
\hline$B C L 11 A$ & ETS1 \\
\hline$C C K$ & ETS1 \\
\hline$C D 38$ & ETS1 \\
\hline$C D K N 2 A$ & ETS1, ELK1 \\
\hline CRISP3 & ETS1 \\
\hline CXCL1 & ETS1 \\
\hline CXCL2 & ETS1 \\
\hline CXCL3 & ETS1, ELK1 \\
\hline CXCL6 & ETS1, ELK1 \\
\hline CXCL9 & ETS1 \\
\hline CXCL10 & ETS1 \\
\hline CXCL11 & ETS1 \\
\hline$D M B T 1$ & ETS1 \\
\hline$F G R$ & ETS1, ELK1 \\
\hline HDAC9 & ETS1 \\
\hline IDOI & ETS1, ELK1 \\
\hline$I L 2 R A$ & ETS1, ELK1 \\
\hline IL5 & ETS1, ELK1 \\
\hline ITGA4 & ETS1 \\
\hline$L T F$ & ETS1 \\
\hline NOS2 & ETS1 \\
\hline$N O X 1$ & ETS1 \\
\hline ORMI & ETS1 \\
\hline PGLYRP1 & ETS1 \\
\hline$P R L R$ & ETS1 \\
\hline$S A A 1$ & ETS1 \\
\hline$S A A 4$ & ETS1 \\
\hline$S H 2 D 1 A$ & ETS1 \\
\hline
\end{tabular}

Table XIV. Genes reported to be targets of ETS family members.

\begin{tabular}{ll}
\hline Gene Name & ETS family members \\
\hline IL5 & ETS1, ETS2, SPI1 \\
ITGA4 & ETS1 \\
LTF & ETS1, SPI1 \\
NOS2 & ELF3 \\
SH2D1A & ETS1, ETS2 \\
\hline
\end{tabular}

Therefore, identifying immunity-related genes in prostate cancer is of a great importance, as well as identifying their potential regulators. Activation and repression of immunityrelated genes have been reported to involve members of the ETS family of transcription factors, as well as the regulation of the immune system (19).

In this study, we report the identification of 37 immunityrelated genes in prostate cancer, and investigated their potential regulation by members of the ETS family of transcription 
factors. We analyzed the expression profile of genes with more than a 10-fold increase or decrease in moderately and poorly differentiated prostate carcinoma glands compared to normal glands of the prostate proper (or the peripheral zone) using two microarrays, and a clustering analysis based on gene function placed each gene into an immunity-related category: B cell, innate and/or T cell immunity. We then searched for ETS transcription factor binding sites within the promoter regions of the 37 genes using TFSEARCH $(33,34)$, and whether any of these genes have been reported to be a target of ETS family members using the TRED database (32).

In addition to the role that most of these genes play in the immune response, many have been implicated in a variety of cancers, including prostate cancer. However, it is not always clear from the findings published in the literature which precise functions these genes play in the network of cellular and molecular mechanisms of antitumor immunology according to current models. Furthermore, in the present study, we have not attempted to evaluate the expression of these genes in immune cells, which may provide further insights of their roles in cancer immunology.

$B$ cell immunity. Our results show that $H D A C 9, C D K N 2 A$, $S H 2 D 1 A$, and $I L 5$ are up-regulated in prostate carcinoma glands compared to the normal glands (Tables I and II). Examination of these genes revealed that most of them are implicated in a variety of cancers, including prostate cancer. Briefly, investigation of human $H D A C$ family members in primary medulloblastoma samples revealed high HDAC9 expression, which was significantly associated with poor survival, and a knockdown of HDAC9 in medulloblastoma cells lead to a decrease in cell growth and viability (39). Other studies have also indicated that high expression HDAC9 is associated with poor prognosis in childhood acute lymphoblastic leukemia (40). A variant of the $C D K N 2 A$ gene has been suggested to be associated with an increased risk of malignant melanoma development (41). SH2D1A is suggested to be involved in the regulation of B cell differentiation (42). Host-derived IL5 has been implicated in promoting experimental malignant pleural effusions and suggested to be involved in its pathogenesis (43).

Analysis of the promoter regions of these genes for potential ETS factor binding sites revealed that $H D A C 9, C D K N 2 A$, SH2DIA and IL5 have binding sites for ETS1, ETS1/ELK1, ETS1 and ETS1/ELK1, respectively (Table XIII) $(33,34)$. SH2DIA and IL5 have been reported to be targets of the ETS factors ETS1/ETS2, and ETS1/ETS2/SPI1, respectively (32,35-38).

On the other hand, the genes LTF, DMBTI, CD38, BCL11A and ITGA4 were found to be down-regulated in prostate carcinoma glands compared to normal glands (Tables VI and VIII). Strikingly, our results show that $L T F$ is 100 -fold down-regulated in prostate carcinoma glands compared to the normal glands, and in agreement with our findings, others have also reported that $L T F$ was the most significantly down-regulated gene in prostate cancer cells (44). DMBT1 is a commonly believed tumor-suppressor gene, that has been suggested to be involved in the carcinogenesis of the malignant brain tumors medulloblastoma and glibolastoma multiforme (45). Furthermore, the $D M B T 1$ gene is also reported to be highly unstable in cancer, and to be involved in the immune defense (46). It has been reported that decreased levels of CD38 are found in prostate adenocarcinoma cells (47). BCL11A is suggested to be involved in lymphoid malignancies (48). ITGA4 has been suggested to be a suppressor of metastasis, as blocking it enhances cell migration in oral squamous cell carcinoma (49).

Analysis for potential ETS factors binding sites within the promoter regions of $L T F, D M B T 1, C D 38, B C L 11 A$ and ITGA4, showed that all of these genes have a potential ETS1 binding site (Table XIII) (33,34). LTF and ITGA4 have been reported to be targets of the ETS factors ETS1/SPI1, and ETS1, respectively $(32,50-52)$.

Innate immunity. Our results show that CRISP3, CXCL11, ADORA1, CXCL9, CXCL10, IDO1, IL2RA, HDAC9, SH2D1A, PGLYRPI, ALOX15, FGR and IL5 are up-regulated in prostate carcinoma glands compared to the normal glands (Tables III and IV). Most of these genes are implicated in a variety of cancers, including prostate cancer. Briefly, others have also reported that CRISP3 is overexpressed in prostate cancer, and suggested that it may serve as a potential biomarker for the disease (53). Other studies also suggested that CRISP3 is an independent predictor of recurrence following radical prostatectomy for localized prostate cancer (54). It has been reported that the chemokines $C X C L 9,10$ and 11 were found to be significantly up-regulated in basal cell carcinoma tissue samples compared with non-lesional skin epithelium (55). Furthermore, high levels of CXCL9 and CXL10 are reported to be secreted in all and in most melanoma metastases, respectively, by tumor endothelial cells (56). ADORAl is found to be up-regulated in breast cancer cell lines, suggesting a role in tumor cell growth and survival (57). Furthermore, the ADORA1 protein expression is found to be higher in most human primary breast tumor tissues compared to normal tissues (57). It has been reported that inhibition of $I D O 1$ promotes growth of T and natural killer cells, leads to IFN- $\gamma$ production increase, and reduction in conversion to regulatory T-like cells (58). It is further suggested that selective inhibition of $I D O I$ effectively regulates mediators of antitumor immunity, making it a potential therapeutic target (58). IL2RA is expressed in various types of cancers, including prostate cancer. It has been reported that high expression of IL2RA in tumors correlates with poor prognosis (59). Peptidoglycan recognition proteins are implicated in innate immunity and anti-cancer defense and PGLYRP1 is reported to play a role in innate immunity (60-62). ALOX15 is reported to have a higher expression in human prostate tumors compared to normal adjacent tissue, and a correlation between ALOX15 and the Gleason-score was reported (63). Additionally, a protumorigenic role for $A L O X 15$ has been suggested in prostate tumor development (63-65). In an ovarian carcinoma study, FGR has been suggested to play a significant role in cancer growth (66). Lastly, HDAC9, SH2DIA and IL5 were discussed in the previous section above.

Analysis for potential ETS factors binding sites within the promoter regions revealed only ETS1 binding sites in CRISP3, CXCL11, ADORA1, CXCL9, CXCL10 and PGLYRPI, but both ETS1/ELK1 binding sites in IDOI, IL2RA and FGR (Table XIII) $(33,34)$.

On the other hand, $L T F, C X C L 2, O R M 1, C C K, C X C L 3$, ORM2, DMBT1, SAA1, C5AR1, SERPINA3, SCGB1A1, CXCL6, SAA4, CXCL1, NOS2, NOX1, AOX1, ACE2 and 
$A B C C 9$ were found to be more than a 10 -fold down-regulated in prostate carcinoma glands compared to the normal glands (Tables IX and X).

It was suggested that malignancy in prostate cancer could be associated with elevated synthesis of angiogenesis stimulating CXC chemokines (67). ORM is suggested to be involved in the protection of tumor cells against immunological attack. $O R M$ was reported to be significantly increased in patients with various types of carcinomas, and was suggested to influence cancer progression via its immunosuppressive properties (68). Furthermore, ORM has been reported to be significantly increased in urine of urinary bladder cancer patients, especially, in patients with invasive tumor, and indications exist that tissue-resident inflammatory cells may play a role in the increase in $O R M$ in addition to the cancer cells (69). Growth of human pancreatic adenocarcinoma has been suggested to depend on $C C K$, and it has been reported that a CCK receptor antagonist inhibits growth of human pancreatic cancer cells in tissue culture and in nude mice (70). It has been reported that during the acute phase response, SAA may be involved in enhancing the migration of monocytes and granulocytes into inflamed tissues (71). During tumor development, location and timing of SAA1 production could define an immune systemevasion strategy for the tumors (72). SAA (SAA1 and SAA2) have been found to be highly expressed in lung cancer tissue (73). Furthermore, $S A A l$ has been found to be overexpressed in ovarian carcinoma tissues, and cell lines, and ovarian carcinoma patients exhibit high SAA serum levels (74). C5AR is expressed on various cells and specifically on the surface of immune cells, and pro-inflammatory polypeptides binds to it (75). High expression level of SERPINA3 has been reported in HLA-positive cervical carcinoma, while in breast cancer tissues, SERPINA3 was found to be down-regulated $(76,77)$. $S C G B 1 A 1$ loss of expression has been reported in prostate cancer and has been suggested to be a possible indicator of disease progression, and NOS 2 has been implicated and correlated with different prostatic diseases including prostate cancer $(78,79)$. NOX1 was found to have increased levels in most human prostate tumor samples, and there are indications that the increase correlates with increased tumorigenicity (80). Additionally, NOX1 protein overexpression is suggested to be an early event in the development of prostate cancer (81). $A O X 1$ is reported to be down-regulated in meningiomas, and hypermethylated in colorectal carcinomas $(82,83)$. ACE2 is reported to be down-regulated in renal tumors, and in pancreatic ductal adenocarcinoma $(84,85)$. The ATP-binding cassette $A B B C C 8$ was found to be amplified in resistant cancer cell lines in a study aiming at investigating chemoresistance (86). Lastly, $L T F$ and DMBT1 were discussed in the previous section above.

Analysis for potential ETS factor binding sites within the promoter regions revealed only ETS1 binding sites in $C X C L 1$, CXCL2, ORM1, CCK, SAA4, NOS2, NOX1, AOX1 and $A B C C 9$, whereas both ETS1/ELK1 binding sites were present in CXCL3 and CXCL6 (Table XIII) $(33,34)$. Additionally, the NOS 2 promoter has been reported to be activated by the ETS factor ELF3 (19).

$T$ cell immunity. The genes IDOI, IL2RA and CDKN2A that were found to be up-regulated in the carcinoma glands and the down-regulated genes $S C G B 1 A 1$ and $B C L 11 A$ have been previously discussed.

PRLR, a member of the cytokine receptor superfamily, which we found to be down-regulated, and PRL are reported to be expressed in both normal and malignant human prostates (87). PRL is recognized as a survival factor involved in the support of tumor growth and chemoresistance in prostate as well as breast cancer (87). PRL is reported to induce apoptosis in androgen-sensitive prostate cancer cells, but not in androgen-insensitive cells, leading to the suggestion that androgen responsiveness could be needed for PRL to be effective (88). Finally, our analysis of the $P R L R$ promoter region, revealed a potential ETS1 binding site within the promoter.

The role of the immune response in tumor progression, and disease outcome is still in dispute (5), and there is a lack of knowledge of the immune defenses in prostate cancer in particular. Thus our study identified a set of 37 immunity-related genes ( $\mathrm{B}$ cell innate and $\mathrm{T}$ cell immunity) that were found to be up or down-regulated in tumors (moderately and poorly differentiated) compared to normal glands of prostate carcinoma patients. We found that in addition to the role that most of these genes may play in the immune response, most have been implicated in a variety of cancers, including prostate cancer. Some of the genes have been reported to be down-regulated in one type of cancer, while up-regulated in another, for example in prostate cancer. These observations may indicate that some of these genes possess unique functions that are specifically related to the type of cancer, as is the case here for prostate cancer, and knowledge of their regulation may shed more light on the role of these genes in the immune response in prostate cancer. In this study, we did not evaluate the expression of these genes in immune cells which may provide further insight into their roles in the immunology of prostate cancer.

We found that $31 / 37$ of these genes have potential binding sites within their promoter regions for members of the ETS family of transcription factors, which have already been reported to be involved in activation or repression of immunity-related genes (Table XIII) (19). Interestingly, all of the 31 genes have potential binding site for ETS1. ETS1, the prototype of the ETS family, is reported to play a key role in immune cell development, and the lack of ETS1 leads to a reduction of T lymphocyte numbers, and to abnormal B lymphocyte differentiation (19). Additionally, ETS1 is reported to be required for the development of natural killer cells (NK) and NK T cells, which are involved in the body's innate immunity defense against malignant cells (19,89-93). Further analysis of these genes revealed that $5 / 31$ genes are reported to be targets of ETS family members (Table XIV) (19,32,35-38,50-52).

An observation that is worth noting is that almost all the genes that overlap between the moderately and the poorly differentiated tumors follow a trend, in which up-regulation or down-regulation in the moderately differentiated tumors is followed by an even higher up or down-regulation in the poorly differentiated tumors, respectively. This observation indicates that these genes may be part of the prostate tumor progression cascade, as the tumor progresses from moderately to poorly differentiated. It may perhaps also provide some hints into the immune response associated with these genes during prostate tumor progression, as well as identifying roles in immunosuppression, protection of tumor cells, or countering the tumor 
cells. The identification and the potential regulation of these immunity-related genes in prostate cancer by ETS factors may provide a better insight into understanding prostate cancer immunology.

\section{Acknowledgements}

We are grateful to the DFG (Deutsche Forschungsgemeinschaft; German Research Association, grant no. WE 1104/11-1, and the Deutsche Krebshilfe (German Cancer Aid, grant no. 107827) for financial support.

\section{References}

1. Kumar-Sinha C, Tomlins SA and Chinnaiyan AM: Recurrent gene fusions in prostate cancer. Nat Rev Cancer 8: 497-511, 2008.

2. Richardson AM, Woodson K, Wang Y, et al: Global expression analysis of prostate cancer-associated stroma and epithelia. Diagn Mol Pathol 16: 189-197, 2007.

3. Arora R, Koch MO, Eble JN, Ulbright TM, Li L and Cheng L: Heterogeneity of Gleason grade in multifocal adenocarcinoma of the prostate. Cancer 100: 2362-2366, 2004

4. Ruijter ET, van de Kaa CA, Schalken JA, Debruyne FM and Ruiter DJ: Histological grade heterogeneity in multifocal prostate cancer. Biological and clinical implications. J Pathol 180: 295-299, 1996.

5. Hsu DS, Kim MK, Balakumaran BS, et al: Immune signatures predict prognosis in localized cancer. Cancer Invest 28: 765-773, 2010.

6. Lin WW and Karin M: A cytokine-mediated link between innate immunity, inflammation, and cancer. J Clin Invest 117: 1175-1183, 2007.

7. Webster WS, Small EJ, Rini BI and Kwon ED: Prostate cancer immunology: biology, therapeutics, and challenges. J Clin Oncol 23: 8262-8269, 2005 .

8. Nelson WG, De Marzo AM, DeWeese TL and Isaacs WB: The role of inflammation in the pathogenesis of prostate cancer. J Urol 172: 6-12, 2004.

9. Poutahidis T, Rao VP, Olipitz W, et al: $\mathrm{CD}^{+}$lymphocytes modulate prostate cancer progression in mice. Int J Cancer 125: 868-878, 2009

10. Shimura S, Yang G, Ebara S, Wheeler TM, Frolov A and Thompson TC: Reduced infiltration of tumor-associated macrophages in human prostate cancer: association with cancer progression. Cancer Res 60: 5857-5861, 2000.

11. Vesalainen S, Lipponen P, Talja M and Syrjanen K: Histological grade, perineural infiltration, tumour-infiltrating lymphocytes and apoptosis as determinants of long-term prognosis in prostatic adenocarcinoma. Eur J Cancer 30A: 1797-1803, 1994

12. Pellat-Deceunynck C: Tumour-associated antigens in multiple myeloma. Br J Haematol 120: 3-9, 2003.

13. Minenkova O, Pucci A, Pavoni E, et al: Identification of tumorassociated antigens by screening phage-displayed human cDNA libraries with sera from tumor patients. Int J Cancer 106: 534-544, 2003.

14. Stockert E, Jager E, Chen YT, et al: A survey of the humoral immune response of cancer patients to a panel of human tumor antigens. J Exp Med 187: 1349-1354, 1998.

15. Bradford TJ, Wang X and Chinnaiyan AM: Cancer immunomics: using autoantibody signatures in the early detection of prostate cancer. Urol Oncol 24: 237-242, 2006.

16. Rajarubendra N, Lawrentschuk N, Bolton DM, Klotz L and Davis ID: Prostate cancer immunology - an update for Urologists. BJU Int 107: 1046-1051, 2011.

17. Joshua AM, Evans A, van der Kwast T, et al: Prostatic preneoplasia and beyond. Biochim Biophys Acta 1785: 156-181, 2008.

18. Cai C, Hsieh CL, Omwancha J, et al: ETV1 is a novel androgen receptor-regulated gene that mediates prostate cancer cell invasion. Mol Endocrinol 21: 1835-1846, 2007.

19. Gallant S and Gilkeson G: ETS transcription factors and regulation of immunity. Arch Immunol Ther Exp 54: 149-163, 2006.

20. Hollenhorst PC, Jones DA and Graves BJ: Expression profiles frame the promoter specificity dilemma of the ETS family of transcription factors. Nucleic Acids Res 32: 5693-5702, 2004.

21. Wasylyk B, Hahn SL and Giovane A: The Ets family of transcription factors. Eur J Biochem 211: 7-18, 1993.
22. Hollenhorst PC, Shah AA, Hopkins C and Graves BJ: Genomewide analyses reveal properties of redundant and specific promoter occupancy within the ETS gene family. Genes Dev 21: 1882-1894, 2007.

23. Wang CY, Petryniak B, Ho IC, Thompson CB and Leiden JM: Evolutionarily conserved Ets family members display distinct DNA binding specificities. J Exp Med 175: 1391-1399, 1992.

24. Oikawa T: ETS transcription factors: possible targets for cancer therapy. Cancer Sci 95: 626-633, 2004.

25. Hashiya N, Jo N, Aoki M, et al: In vivo evidence of angiogenesis induced by transcription factor Ets-1: Ets-1 is located upstream of angiogenesis cascade. Circulation 109: 3035-3041, 2004.

26. Rothhammer T, Hahne JC, Florin A, et al: The Ets-1 transcription factor is involved in the development and invasion of malignant melanoma. Cell Mol Life Sci 61: 118-128, 2004.

27. Hahne JC, Okuducu AF, Kaminski A, Florin A, Soncin F and Wernert N: Ets-1 expression promotes epithelial cell transformation by inducing migration, invasion and anchorage-independent growth. Oncogene 24: 5384-5388, 2005.

28. He J, Pan Y, Hu J, Albarracin C, Wu Y and Dai JL: Profile of Ets gene expression in human breast carcinoma. Cancer Biol Ther 6: 76-82, 2007.

29. Wellmann A, Wollscheid V, Lu H, et al: Analysis of microdissected prostate tissue with ProteinChip ${ }^{\circledR}$ arrays - a way to new insights into carcinogenesis and to diagnostic tools. Int J Mol Med 9: 341-347, 2002.

30. Okuducu AF, Janzen V, Hahne JC, Ko Y and Wernert N: Influence of histochemical stains on quantitative gene expression analysis after laser-assisted microdissection. Int J Mol Med 11: 449-453, 2003.

31. Okuducu AF, Hahne JC, Von Deimling A and Wernert N: Laser-assisted microdissection, techniques and applications in pathology (Review). Int J Mol Med 15: 763-769, 2005.

32. Cold Spring Harbor Laboratory: Transcriptional Regulatory Element Database, available at http://rulai.cshl.edu/cgi-bin/ TRED/tred .cgi?process=home, accessed in 2011 .

33. TFSEARCH: Searching Transcription Factor Binding Sites. Available at http://www.cbrc.jp/research/db/TFSEARCH.html accessed in 2011 .

34. Heinemeyer T, Wingender E, Reuter I, et al: Databases on transcriptional regulation: TRANSFAC, TRRD and COMPEL. Nucleic Acids Res 26: 362-367, 1998.

35. Okamoto S, Ji H, Howie D, et al: Expression of the SH2D1A gene is regulated by a combination of transcriptional and posttranscriptional mechanisms. Eur J Immunol 34: 3176-3186, 2004

36. Blumenthal SG, Aichele G, Wirth T, Czernilofsky AP, Nordheim A and Dittmer J: Regulation of the human interleukin-5 promoter by Ets transcription factors. Ets1 and Ets2, but not Elf-1, cooperate with GATA3 and HTLV-I Tax1. J Biol Chem 274: 12910-12916, 1999.

37. van Dijk TB, Baltus B, Raaijmakers JA, Lammers JW, Koenderman L and de Groot RP: A composite C/EBP binding site is essential for the activity of the promoter of the IL3/IL5/ granulocyte-macrophage colony-stimulating factor receptor beta c gene. J Immunol 163: 2674-2680, 1999.

38. van Dijk TB, Baltus B, Caldenhoven E, et al: Cloning and characterization of the human interleukin-3 (IL3)/IL-5/ granulocyte-macrophage colony-stimulating factor receptor betac gene: regulation by Ets family members. Blood 92: 3636-3646, 1998.

39. Milde T, Oehme I, Korshunov A, et al: HDAC5 and HDAC9 in medulloblastoma: novel markers for risk stratification and role in tumor cell growth. Clin Cancer Res 16: 3240-3252, 2010.

40. Moreno DA, Scrideli CA, Cortez MA, et al: Differential expression of HDAC3, HDAC7 and HDAC9 is associated with prognosis and survival in childhood acute lymphoblastic leukaemia. Br J Haematol 150: 665-673, 2010.

41. Debniak T, Scott RJ, Huzarski T, et al: CDKN2A common variants and their association with melanoma risk: a population-based study. Cancer Res 65: 835-839, 2005.

42. Mikhalap SV, Shlapatska LM, Yurchenko OV, et al: The adaptor protein SH2D1A regulates signaling through CD150 (SLAM) in B cells. Blood 104: 4063-4070, 2004.

43. Stathopoulos GT, Sherrill TP, Karabela SP, et al: Host-derived interleukin-5 promotes adenocarcinoma-induced malignant pleural effusion. Am J Respir Crit Care Med 182: 1273-1281, 2010.

44. Shaheduzzaman S, Vishwanath A, Furusato B, et al: Silencing of lactotransferrin expression by methylation in prostate cancer progression. Cancer Biol Ther 6: 1088-1095, 2007.

45. Mollenhauer J, Wiemann S, Scheurlen W, et al: DMBT1, a new member of the SRCR superfamily, on chromosome 10q25.3-26.1 is deleted in malignant brain tumours. Nat Genet 17: 32-39, 1997. 
46. Mollenhauer J, Herbertz S, Holmskov U, et al: DMBT1 encodes a protein involved in the immune defense and in epithelial differentiation and is highly unstable in cancer. Cancer Res 60: 1704-1710, 2000.

47. Liu AY, Roudier MP and True LD: Heterogeneity in primary and metastatic prostate cancer as defined by cell surface $\mathrm{CD}$ profile. Am J Pathol 165: 1543-1556, 2004.

48. Satterwhite E, Sonoki T, Willis TG, et al: The BCL11 gene family: involvement of BCL11A in lymphoid malignancies. Blood 98: 3413-3420, 2001.

49. Zhang Y, Lu H, Dazin P and Kapila Y: Functional differences between integrin $\alpha 4$ and integrins $\alpha 5 / \alpha \mathrm{v}$ in modulating the motility of human oral squamous carcinoma cells in response to the $V$ region and heparin-binding domain of fibronectin. Exp Cell Res 295: 48-58, 2004.

50. Liu D, Wang X, Zhang Z and Teng CT: An intronic alternative promoter of the human lactoferrin gene is activated by Ets. Biochem Biophys Res Commun 301: 472-479, 2003.

51. Teng CT, Liu Y, Yang N, Walmer D and Panella T: Differential molecular mechanism of the estrogen action that regulates lactoferrin gene in human and mouse. Mol Endocrinol 6: 1969-1981, 1992.

52. Rosen GD, Barks JL, Iademarco MF, Fisher RJ and Dean DC: An intricate arrangement of binding sites for the Ets family of transcription factors regulates activity of the $\alpha 4$ integrin gene promoter. J Biol Chem 269: 15652-15660, 1994.

53. Kosari F, Asmann YW, Cheville JC and Vasmatzis G: Cysteinerich secretory protein-3: a potential biomarker for prostate cancer. Cancer Epidemiol Biomarkers Prev 11: 1419-1426, 2002.

54. Bjartell AS, Al-Ahmadie H, Serio AM, et al: Association of cysteine-rich secretory protein 3 and beta-microseminoprotein with outcome after radical prostatectomy. Clin Cancer Res 13: 4130-4138, 2007.

55. Lo BK, Yu M, Zloty D, Cowan B, Shapiro J and McElwee KJ: CXCR3/ligands are significantly involved in the tumorigenesis of basal cell carcinomas. Am J Pathol 176: 2435-2446, 2010

56. Amatschek S, Lucas R, Eger A, et al: CXCL9 induces chemotaxis, chemorepulsion and endothelial barrier disruption through CXCR3-mediated activation of melanoma cells. Br J Cancer 104 469-479, 2011

57. Mirza A, Basso A, Black S, et al: RNA interference targeting of A1 receptor-overexpressing breast carcinoma cells leads to diminished rates of cell proliferation and induction of apoptosis. Cancer Biol Ther 4: 1355-1360, 2005.

58. Liu X, Shin N, Koblish HK, et al: Selective inhibition of IDO1 effectively regulates mediators of antitumor immunity. Blood 115: 3520-3530, 2010

59. Kuhn DJ and Dou QP: The role of interleukin-2 receptor $\alpha$ in cancer. Front Biosci 10: 1462-1474, 2005.

60. Sashchenko LP, Dukhanina EA, Yashin DV, et al: Peptidoglycan recognition protein tag7 forms a cytotoxic complex with heat shock protein 70 in solution and in lymphocytes. J Biol Chem 279: 2117-2124, 2004

61. Kang D, Liu G, Lundstrom A, Gelius E and Steiner H: A peptidoglycan recognition protein in innate immunity conserved from insects to humans. Proc Natl Acad Sci USA 95: 10078-10082, 1998.

62. Cho JH, Fraser IP, Fukase K, et al: Human peptidoglycan recognition protein $\mathrm{S}$ is an effector of neutrophil-mediated innate immunity. Blood 106: 2551-2558, 2005.

63. Kelavkar UP, Cohen C, Kamitani H, Eling TE and Badr KF Concordant induction of 15-lipoxygenase-1 and mutant p53 expression in human prostate adenocarcinoma: correlation with Gleason staging. Carcinogenesis 21: 1777-1787, 2000.

64. Hsi LC, Wilson LC and Eling TE: Opposing effects of 15-lipoxygenase-1 and -2 metabolites on MAPK signaling in prostate. Alteration in peroxisome proliferator-activated receptor gamma. J Biol Chem 277: 40549-40556, 2002.

65. Kelavkar UP, Nixon JB, Cohen C, Dillehay D, Eling TE and Badr KF: Overexpression of 15-lipoxygenase-1 in PC-3 human prostate cancer cells increases tumorigenesis. Carcinogenesis 22: $1765-1773,2001$.

66. Kim HS, Han HD, Armaiz-Pena GN, et al: Functional roles of Src and Fgr in ovarian carcinoma. Clin Cancer Res 17: 1713-1721, 2011.

67. Engl T, Relja B, Blumenberg C, et al: Prostate tumor CXCchemokine profile correlates with cell adhesion to endothelium and extracellular matrix. Life Sci 78: 1784-1793, 2006.

68. Fan C, Stendahl U, Stjernberg N and Beckman L: Association between orosomucoid types and cancer. Oncology 52: 498-500, 1995.
69. Irmak S, Tilki D, Heukeshoven J, et al: Stage-dependent increase of orosomucoid and zinc- $\alpha 2$-glycoprotein in urinary bladder cancer. Proteomics 5: 4296-4304, 2005.

70. Funakoshi A and Kono A: Growth inhibition of human pancreatic cancer cells by cholecystokinin receptor antagonist in tissue culture and in nude mice. Gastroenterol Jpn 27: 78-82, 1992.

71. Badolato R, Wang JM, Murphy WJ, et al: Serum amyloid A is a chemoattractant: induction of migration, adhesion, and tissue infiltration of monocytes and polymorphonuclear leukocytes. J Exp Med 180: 203-209, 1994

72. Mattarollo SR and Smyth MJ: A novel axis of innate immunity in cancer. Nat Immunol 11: 981-982, 2010

73. Sung HJ, Ahn JM, Yoon YH, et al: Identification and validation of SAA as a potential lung cancer biomarker and its involvement in metastatic pathogenesis of lung cancer. J Proteome Res 10: 1383-1395, 2011.

74. Urieli-Shoval S, Finci-Yeheskel Z, Dishon S, et al: Expression of serum amyloid a in human ovarian epithelial tumors: implication for a role in ovarian tumorigenesis. J Histochem Cytochem 58: 1015-1023, 2010

75. Monk PN, Scola AM, Madala P and Fairlie DP: Function, structure and therapeutic potential of complement C5a receptors. Br J Pharmacol 152: 429-448, 2007.

76. Kloth JN, Gorter A, Fleuren GJ, et al: Elevated expression of SerpinA1 and SerpinA3 in HLA-positive cervical carcinoma. J Pathol 215: 222-230, 2008.

77. Cimino D, Fuso L, Sfiligoi C, et al: Identification of new genes associated with breast cancer progression by gene expression analysis of predefined sets of neoplastic tissues. Int J Cancer 123 : 1327-1338, 2008.

78. Weeraratna AT, Cajigas JA, Schwartz A, Enquist EG, Manyak MJ and Patierno SR: Loss of uteroglobin expression in prostate cancer: relationship to advancing grade. Clin Cancer Res 3: 2295-2300, 1997.

79. Yoo KH, Kim SK, Chung JH and Chang SG: Nitric oxide synthase 2 gene polymorphisms are associated with prostatic volume in Korean men with benign prostatic hyperplasia. Asian J Androl 12: 690-696, 2010

80. Lim SD, Sun C, Lambeth JD, et al: Increased Nox1 and hydrogen peroxide in prostate cancer. Prostate 62: 200-207, 2005.

81. Arnold RS, He J, Remo A, et al: Nox1 expression determines cellular reactive oxygen and modulates c-fos-induced growth factor, interleukin-8, and Cav-1. Am J Pathol 171: 2021-2032, 2007.

82. Fevre-Montange M, Champier J, Durand A, et al: Microarray gene expression profiling in meningiomas: Differential expression according to grade or histopathological subtype. Int J Oncol 35: 1395-1407, 2009.

83. Oster B, Thorsen K, Lamy P, et al: Identification and validation of highly frequent $\mathrm{CpG}$ island hypermethylation in colorectal adenomas and carcinomas. Int J Cancer: Mar 11, 2011 (Epub ahead of print).

84. Larrinaga G, Perez I, Sanz B, et al: Angiotensin-converting enzymes (ACE and ACE2) are downregulated in renal tumors. Regul Pept 165: 218-223, 2010.

85. Zhou L, Zhang R, Yao W, et al: Decreased expression of angiotensin-converting enzyme 2 in pancreatic ductal adenocarcinoma is associated with tumor progression. Tohoku J Exp Med 217: 123-131, 2009

86. Yasui K, Mihara S, Zhao C, et al: Alteration in copy numbers of genes as a mechanism for acquired drug resistance. Cancer Res 64: 1403-1410, 2004

87. Jacobson EM,Hugo ER, Tuttle TR,Papoian R and Ben-Jonathan N: Unexploited therapies in breast and prostate cancer: blockade of the prolactin receptor. Trends Endocrinol Metab 21: 691-698, 2010.

88. Giuffrida D, Perdichizzi A, Giuffrida MC, et al: Does prolactin induce apoptosis? Evidences in a prostate cancer in vitro model. J Endocrinol Invest 33: 313-317, 2010.

89. Barton K, Muthusamy N, Fischer C, et al: The Ets-1 transcription factor is required for the development of natural killer cells in mice. Immunity 9: 555-563, 1998.

90. Walunas TL, Wang B, Wang CR and Leiden JM: Cutting edge: the Ets1 transcription factor is required for the development of NK T cells in mice. J Immunol 164: 2857-2860, 2000.

91. Biron CA and Brossay L: NK cells and NKT cells in innate defense against viral infections. Curr Opin Immunol 13: 458-464, 2001.

92. Korbel DS, Finney OC and Riley EM: Natural killer cells and innate immunity to protozoan pathogens. Int J Parasitol 34: 15171528,2004

93. Wu J and Lanier LL: Natural killer cells and cancer. Adv Cancer Res 90: 127-156, 2003. 